\title{
Simplified Large-Area Manufacturing of Organic Electrochemical Transistors Combining Printing and a Self-Aligning Laser Ablation Step
}

Thomas Blaudeck, Peter Andersson Ersman, Mats Sandberg, Sebastian Heinz, Ari Laiho,

Jiang Liu, Isak Engquist, Magnus Berggren and Reinhard R. Baumann

\section{Linköping University Post Print}

N.B.: When citing this work, cite the original article.

This is the authors' version of the original publication:

Thomas Blaudeck, Peter Andersson Ersman, Mats Sandberg, Sebastian Heinz, Ari Laiho, Jiang Liu, Isak Engquist, Magnus Berggren and Reinhard R. Baumann, Simplified LargeArea Manufacturing of Organic Electrochemical Transistors Combining Printing and a SelfAligning Laser Ablation Step, 2012, Advanced Functional Materials, (22), 14, 2939-2948.

http://dx.doi.org/10.1002/adfm.201102827

Copyright: Wiley-VCH Verlag Berlin http://www.wiley-vch.de/publish/en/

Postprint available at: Linköping University Electronic Press http://urn.kb.se/resolve?urn=urn:nbn:se:liu:diva-79665 
Version: 2012-02-20

\section{Simplified large-area manufacturing of organic electrochemical transistors combining printing and a self-aligning laser ablation step}

Thomas Blaudeck ${ }^{\mathrm{a}, \mathrm{b}, \mathrm{c}}$, Peter Andersson Ersman ${ }^{\mathrm{d}}$, Mats Sandberg $^{\mathrm{d}}$, Sebastian Heinz ${ }^{\mathrm{b}}$, Ari Laiho ${ }^{\mathrm{a}, \mathrm{e}}$, Jiang Liu ${ }^{\mathrm{a}}$, Isak Engquist ${ }^{\mathrm{a}}$, Magnus Berggren ${ }^{\mathrm{a}}$, Reinhard R. Baumann ${ }^{\mathrm{b}, \mathrm{f}}$

${ }^{a}$ Linköping University, Department of Science and Technology (ITN), Organic Electronics, 60174 Norrköping, Sweden

${ }^{b}$ Chemnitz University of Technology, Institute for Print and Media Technology, 09107 Chemnitz, Germany

${ }^{c}$ Chemnitz University of Technology, Center for Microtechnologies, 09107 Chemnitz, Germany

${ }^{d}$ Acreo AB, Printed Electronics, 60221 Norrköping, Sweden

${ }^{e}$ Aalto University School of Science and Technology, Advanced Magnetic Imaging Centre, 02150 Espoo, Finland

${ }^{f}$ Fraunhofer Institute for Electronic Nanosystems (ENAS), Department Printed Functionalities, 09126 Chemnitz, Germany

Corresponding author: Magnus Berggren (phone: +46.(0)11.363637, e-mail: magnus.berggren@liu.se) 
Keywords: printed electronics, organic electrochemical transistors, screen printing, laser ablation, inkjet printing

\title{
Table of content:
}

We report on a hybrid sheet-based manufacturing approach for organic electrochemical transistors comprising printing and a self-aligning laser ablation step.

\begin{abstract}
We report on a hybrid manufacturing approach for organic electrochemical transistors (OECTs) on flexible substrates. The technology is based on conventional and digital printing (screen and inkjet printing), laser processing, and post-press technologies. A careful selection of the conductive, dielectric and semiconductor materials with respect to their optical properties enables a self-aligning pattern formation which results in a significant reduction of the usual registration problems during manufacturing. For the prototype OECTs, based on this technology, we obtained on/off ratios up to 600 and switching times of 100 milliseconds at gate voltages in the range of 1 V.
\end{abstract}




\section{Introduction}

Manufacturing of printed transistors has to cope with the challenge to reliably deliver devices exhibiting high on/off-ratios and low operating voltages, preferably below $3 \mathrm{~V}$, enabling for instance the utilization of printed batteries in mobile applications or sensing in aqueous environments. In literature three different approaches are in principle followed: (i) organic fieldeffect transistors (OFETs) ${ }^{/ 1 / 2 / / 3 /}$, (ii) (poly)electrolyte-gated organic field-effect transistors (EGOFETs) $^{/ 4 / / 5 / / 6 / 7 / / 8 /}$ and (iii) organic electrochemical transistors (OECTs). ${ }^{\text {9//10//11/12/13/14/ }}$ For OFETs, reasonably low operating voltages are obtained by using high-k dielectric materials ${ }^{\mathbf{1} /}$ or ultra-thin dielectric layers obtained by self-assembled molecular monolayers. ${ }^{/ 2 /}$ Both OFET approaches have certain drawbacks regarding manufacturing: whereas the former is accompanied by introduction of charge traps, the implementation of the latter by scalable printing methods is limited. When it comes to application of electronics in the low-cost sector, e.g. as added value (sensor, signage element, etc.) on consumer products such as food packages, the costs of the manufacture must not significantly exceed the ones of usual graphically printed items. The requirement for OFETs to have an ultra-short transistor channel implies a reduction of the minimal patterning feature size down to the sub-micrometer scale. This fact increases the equipment costs as it drives the demand for the involved techniques towards and beyond their usual resolution limits. At this point, the OECTs enter the scene. OECTs are typically slower, but operate at voltages in the range of several volts and can run higher currents through the channel as OFETs of similar dimensions. Further, the electrical parameters of OECTs do not strictly relate to the geometrical constraints: the channel is made of a conducting polymer which is in contact with an electrolyte that, in turn, contacts with a gate electrode. ${ }^{/ 11 /}$ The OECT concept enabled freeform fabrication ${ }^{/ \mathbf{1 2} /}$ and solution processing such as spin coating. ${ }^{/ \mathbf{1 3} / \mathbf{1 4} /}$ Still, 
irrespective of the transistor concept, a limiting factor for an upscaling of production, e.g. towards roll-to-roll or sheet-based manufacturing of complex logic device arrays, is the quality of the multilayer registration between the individual process steps. In this paper, we present a hybrid manufacturing approach based on a combination of different printing techniques and laser structuring. The key of our manufacturing concept is the introduction of a self-aligning laserablation process amidst screen printing and inkjet printing passes. The subtractive step allows for vertical OECT device architectures by tremendously reducing the registration demands during manufacturing, resulting in arrays of OECTs $(17 \times 50)$ fabricated on DIN A4 $\left(297 \times 210 \mathrm{~mm}^{2}\right)$ flexible sheet substrates.

In literature, first reports on transistors based on electrochemical switching of organic materials dates back to the $1980 \mathrm{~s},{ }^{/ 15 /}$ followed by various reports on microelectrochemical transistors (micro-ECTs) based on polycarbazole ${ }^{/ 16 /}$ and polyindole ${ }^{/ 17 /}$ using both aqueous and non-aqueous electrolytes. Berggren and co-workers have developed a lateral OECT architecture ${ }^{\text {/9/ }}$ as a prerequisite for all-printed logic circuitry ${ }^{/ \mathbf{1 0} /}$ and all-organic active-matrix displays ${ }^{/ \mathbf{1 8} /}$ achieved with standard printing techniques. Mannerbro et al. reported a fully inkjet-printed ring oscillator, /19/ whereas the work of Kaihovirta et al. relied on flexographic printing of source, drain, and gate electrodes and self-assembly of the electrolyte on a pre-patterned substrate. ${ }^{/ 20 /}$

In electrochemical devices that utilize organic conducting polymers as the active material, both ions and electrons can be charge carriers. In the concept of thin-film OECTs developed earlier, ${ }^{\text {/9/ }}$ electrons serve as the charge carriers, while the ions in the electrolyte enable the electrochemical reactions. As the ions are subject to chemical interaction with their environment and the conductivity of the polymeric materials can be controlled by changing their particular redox state, /17/ OECTs can serve as building blocks for a variety of sensing devices. In contrast to OFETs, which are driven by field effects in channels that are only one or a few to tens of nanometers 
thick, the electronic functionality of OECTs is based on a coupling between ion and electron transport in the bulk material. With that, they are less sensitive to dimensional constraints ${ }^{/ 20 /}$ and qualify potentially for manufacture by printing and other microstructuring techniques that allow for high throughput at limited precision. The concept of OECTs offers unique possibilities for the realization of electronic circuits with non-vacuum fabrication techniques because of their relative insensitivity to precise device geometry. ${ }^{/ 21 /}$ Therefore, this concept was recently adapted in OECTs woven from silk fibers. ${ }^{122 /}$

Zirkl et al. recently reported that the fabrication of OECTs requires the least number of printing passes when choosing a lateral device architecture $\left(1^{\text {st }}\right.$ : bottom electrodes, $2^{\text {nd }}:$ source, drain and gate electrodes and transistor channel, $3^{\text {rd }}$ : electrolyte). ${ }^{23 /} \mathrm{A}$ reduction of the number of manufacturing steps down to the least possible number is beneficial for the device yield and cost, but only when a fully additive (“all-printed") manufacturing workflow is considered: all-additive devices use expensive materials more efficiently than devices that are made by subtractive techniques, since for the latter ones, there is always some material removed and wasted. In selected cases, the introduction of a subtractive step can have benefits, for instance when vertically stacked devices ${ }^{124 /}$ are envisaged that can be much smaller than lateral ones, reducing the volume of functional material to be printed and reducing switching time. A further argument for such a workflow is that the fraction of wasted costly material becomes smaller when coated area increases so that for large coated areas, the difference compared to all-additive printing is insignificant.

In principle, in a vertical stack structure, the minimum distance between two electrode layers separated by an insulator layer is given by the smallest layer thickness. For practically all solution-based roll-to-roll-compatible deposition techniques such as printing, the minimum achievable layer thickness is much smaller than the minimum lateral feature size. In that respect, 
recent comparative reviews for organic ${ }^{25 /}$ and inorganic $^{/ 26 /}$ functional materials report minimum layer thicknesses of sub- to several micrometers, which should be compared with minimum lateral feature sizes in the range of 10 to 100 micrometers for techniques like doctor blading, spray coating, inkjet printing, and others. In other words, the introduction of subtractive processes into an otherwise additive manufacturing line would enhance the number of OECT design geometries at disposal for various application scenarios, as the device structures can extend to a third dimension. Here, the advantages of the subtractive technique will dominate, for example to achieve smaller (and thereby faster) components. However, the requirements for the additional, non-printing steps are that they can be performed in a high yield and do not require too careful registration issues, meaning in the best case, that they are self-aligning.

The choice of the particular manufacturing techniques is very important. Vertically stacked devices can be achieved with roll-to-roll or sheet-based deposition techniques, but they require the inclusion of subtractive techniques for a patterning of the multilayers after deposition. Reviewing the individual progress in several disciplines of mechanical engineering in recent years, viable concepts for an integration of additive (coating, printing, dispensing) with transformational (surface-energy patterning ${ }^{/ 27 /}$, sintering ${ }^{/ 28 / 29 /}$, print-and-drag ${ }^{/ 30 /}$, forced drying ${ }^{\mathbf{3 1} /}$ ) and subtractive (roll-to-roll liftoff, ${ }^{\text {/32/ }}$ dry-phase patterning, ${ }^{\text {/33/ }}$ laser ablation ${ }^{\text {/34//35/) }}$ techniques could be developed in the context of roll-to-roll or sheet-fed manufacturing. The hybrid approach is currently discussed in organic and inorganic thin-film photovoltaics, where large-area coated film stacks are patterned via laser ablation into separate individual photovoltaic cells $^{/ 35 /}$. However, when combining such technologies to a hybrid manufacturing line, rigorous requirements regarding the alignment precision are set.

Although there are some good arguments for all-printed device manufacturing without any subtractive techniques for low-cost electronic applications ${ }^{\mathbf{3 1} / / 36 /}$ or photovoltaics ${ }^{/ 37 / / 38 /}$, the 
introduction of subtractive steps can be interesting for large-area formats (e.g. DIN A4). As discussed above, when the totally coated area increases, the relative amount of removed material is small and the benefits of a more versatile or robust device geometry may win over the purely economic fact that some costly material has to be removed.

In printed electronics, first examples for devices based on hybrid manufacturing workflows are the concept of vertical polyelectrolyte-gated organic field-effect transistors (VEGOFETs), ${ }^{\text {/39/ }}$ the above-mentioned all-printed ferroelectric active matrix sensor networks, ${ }^{/ 23 /}$ or a fully integrated roll-to-roll manufacturing scenario for RFID tags on plastic foils. ${ }^{/ 40 /}$ For OECTs, in spite of their "more relaxed" requirements on feature size compared to other transistor concepts, aside of the

work by Tehrani et al. on printable electrochemical circuits ${ }^{\mathbf{4} 1 /}$, such technological development is not yet reported, which serves as the key motivation to this work.

\section{Results and Discussion}

\section{$<$ Figure $1>$}

The top view and side view of the stack which builds the individual OECT is shown in Fig. 1. The self-alignment of the subtractive step is based on a difference in the absorption properties of the source-drain structure (sub-layer) and the dielectric layer. To achieve the stack, Fig. 2 shows the schematic workflow. The process starts with screen printing two passes on a flexible substrate: a conductive layer (which will act as the source-drain structure of the OECT) and a dielectric layer (which thoroughly separates the electrolyte from being in contact with the sourcedrain structure) preventing parasitic leakage currents. Fig. 2 illustrates how the usage of laser 
ablation as a lateral and vertical structuring tool solves the registration issues during the manufacturing process. The laser light causes local heating and evaporation of the conductive layer, which also results in perforation of the dielectric. During this step, the precision in positioning of the individual source-drain structure does not have to be better than the lateral dimensions of the dielectric layer (order of a few millimeters), which is easily achieved even by manual or semi-automatic printing equipment ("by eye") without expensive alignment technology. Microscope images of the components during the fabrication processes, screen printing, laser ablation and inkjet printing, can be found in Fig. 3. A square-shaped channel geometry (Fig. 3B) is aimed for as the optimum with respect to the achievable on/off ratios $\left(\mathrm{I}_{\mathrm{DS}}\left(\mathrm{V}_{\mathrm{G}}=0\right) / \mathrm{I}_{\mathrm{DS}}\left(\mathrm{V}_{\mathrm{G}}=1 \mathrm{~V}\right)=1 \times 10^{3 / 42 /}\right.$.

$<$ Figure 2>

$<$ Figure 3>

An important question is whether the deposited PEDOT:PSS finds the crack-like cavity in the dielectric layer induced by the laser ablation step. From the microscope images (Fig. 3B and Fig. 3D), the hypothesis can be formed that the PEDOT:PSS is driven by capillary forces to the crack to form an effective transistor channel between the two separated parts of the source-drain structure. To shed light on this question, a screen-printed and disconnected component (printed conductive source-drain layer and dielectric layer, after laser irradiation) was cut in half (Fig. 4A) precisely in the region of the laser trajectory (Fig. 4B) and investigated by cross-sectional scanning electron microscopy (SEM) (Fig. 4D) at an angle of inspection of ca. $30^{\circ}$. 
$<$ Figure 4>

The contrast-enhanced grey-scale image in Fig. 4D shows a clear delamination of a platelet of the dielectric layer, giving rise to a cavity formation below this layer. This cavity is in conjectural contact with the remaining parts of the conductive layer. Consulting comparative measurements with two-dimensional optical profilometry (Fig. 4B and Fig. 4C) we quantified a height difference of $6 \mu \mathrm{m}$ next to the formed "crack" in comparison to $3 \mu \mathrm{m}$ at the areas where the dielectric layer covers the conductive layer.

Our understanding of the laser ablation process is as follows: as the dielectric layer and the substrate are fully transparent to the incident laser irradiation, the ablation condition is solely fulfilled at the places where the laser trajectory hits the carbon layer through the dielectric layer. Based on the matching absorption spectrum of the carbon layer with the laser emission wavelength around $1064 \mathrm{~nm},{ }^{/ 23 /}$ the energy is selectively absorbed and dissipated, forming a heat-affected zone. As a consequence, depending on the degree of satisfaction of the ablation threshold condition, the carbon is either melted or evaporated ${ }^{\mathbf{4} 3 /}$, causing it to expand. The transparent dielectric top-layer is therefore delaminated due to the volume expansion after the evaporation of the light-absorbing conductive sub-layer. With respect to the evaporation process, the sudden increase in the volume of the sublimated carbon induces an increase of the local pressure which can be compensated only by the formation of a crack in the dielectric top-layer. When inspecting carefully the optical profilogram in Fig. 4B, one can clearly see that a crack is formed in the dielectric layer more or less exactly parallel to the edge of the printed carbon sub- 
layer. It extends to a region up to $100 \mu \mathrm{m}$ left and right of the incident laser beam along its trajectory. Once this crack is formed, it acts as a "chimney" for the gaseous material released in the successive laser ablation events, where the cavity is enlarged due to the local impact of the evaporated carbon on the dielectric top-layer. A similar effect is known for laser structuring of transparent inorganic materials on opaque flexible substrates ${ }^{144 /}$. Without going into details of the possibly involved phase transitions, the delamination has analogies to the concept of a laserinduced backward transfer (LIBT) recently put forward by Kusnetsov et al. ${ }^{145 /}$ Indeed, preliminary results of a chemical mapping of the channel region of our OECTs with z-scanning depth-profile confocal Raman microscopy (band assignment according to Ref.s /46/ and /47/) indicate that at least traces of carbon are present in the formed cavity, conjecturally at its "ceiling" (the downside of the elevated platelet of the dielectric top-layer) (cf. Supporting Information).

Besides the indication in the cross-sectional SEM (Fig. 4D), the existence of the cavity can be implied from the discrepancy between the individual layer thicknesses of the printed carbon (3-4 $\mu \mathrm{m})$ and the dielectric $(10-13 \mu \mathrm{m})$ layers (cf. Supporting Information): when looking at the profilogram (Fig. 4B) and the height profile (Fig. 4C), one observes that there is an effective elevation of the dielectric layer by $5-6 \mu \mathrm{m}$ which is clearly restricted to the area where the carbon was exposed to the laser beam. A third, but indirect method to probe the dimensions of the transistor channel is to measure the resistivity of the component after the reconnection step. Assuming that the 100-150 $\mu \mathrm{m}$ wide gap in the carbon layer (cf. Fig. 2B) is reconnected by PEDOT:PSS forming a solid film with a resistivity of the order of $10 \mathrm{~S} / \mathrm{cm},{ }^{148 / 49 /}$ the PEDOT:PSS film has to have a minimum effective layer thickness of the order of $0.1-1 \mu \mathrm{m}$ to achieve the measured final resistance of $2-20 \mathrm{k} \Omega$ (Fig. 5A). 
At this point, it is obvious to ask if the amount of carbon which is not evaporated (and hence not carried away through the formed crack) or the quality of the reconnection step are related to the manufacture yield of the disconnection step. In order to answer this question, we performed a large-area sampling on the yield of the laser-assisted disconnection on 153 printed source-drain structures and the yield of the channel formation on another batch of the same number of structures. The results are visualized in Fig. 5.

$<$ Figure 5>

In the upper part of Fig. 5A, the occurrence of resistances measured after the laser disconnection step (cf. Experimental Section) is shown as a function of the applied laser scan speed varied in the range $1500-4500 \mathrm{~mm} / \mathrm{s}$. We considered a source-drain structure disconnected if a resistance of $2 \mathrm{M} \Omega$ or higher was measured, compared to the 5-20 k $\Omega$ measured for the printed carbon structures. For a laser scan speed of $1500 \mathrm{~mm} / \mathrm{s}$ (equals a $7.5 \mu \mathrm{m}$ spacing of the individual laser spots along the trajectory at $200 \mathrm{kHz}$ repetition rate), more than $98 \%$ of all source-drain structures could be considered disconnected, whereas the yield is only $56 \%$ for a laser scan speed of $4500 \mathrm{~mm} / \mathrm{s}(22.5 \mu \mathrm{m}$ spot spacing $)$. The result can be explained by the spot spacing: if the laser spots are placed too far apart from each other, the carbon layer cannot be successfully disconnected along the entire laser trajectory. This result was also observed in control experiments at higher laser scan speeds $(5500 \mathrm{~mm} / \mathrm{s}$ and higher) where a disconnection was not achieved-almost all source-drain structures kept their initial low resistance. Hence, the scan speed is a limiting factor with respect to the achievable manufacturing throughput. 
Additionally, the yield of the reconnection step by inkjet printing is important. In the lower part of Fig. 5A, the results are displayed in the same fashion as for the disconnection step. For the fabrication of OECTs, to introduce a clear threshold, we considered a source-drain structure connected (meaning that it has been successfully reconnected or that it never was disconnected) if a resistance of $200 \mathrm{k} \Omega$ or lower was measured. For a laser scan speed of $1500 \mathrm{~mm} / \mathrm{s}$, only $10 \%$ of the structures achieve resistances below $200 \mathrm{k} \Omega$ after reconnection and the remaining $90 \%$ have to be discarded. For $3500 \mathrm{~mm} / \mathrm{s}$, more than $20 \%$ are (re)connected properly and for 4500 $\mathrm{mm} / \mathrm{s}$, the number is roughly $50 \%$. However, for the latter case of higher laser scan speeds, we know from the disconnection experiments that this number includes structures that were never disconnected so that they cannot be used to make OECTs. Hence, the choice is a compromise: using scan speeds between $2500 \mathrm{~mm} / \mathrm{s}$ and $3500 \mathrm{~mm} / \mathrm{s}$, a total yield of 20-25\% can be reliably obtained for the manufacture of OECTs.

The improvement of the reconnection step could be explained by observing the geometrical shape of the crack measured by optical profilometry as a function of the laser scan speed (Fig. 5B). For the smallest spot spacing $(7.5 \mu \mathrm{m}$ at $1500 \mathrm{~mm} / \mathrm{s})$, the crack has a linear shape, whereas for the highest spot spacing $(22.5 \mu \mathrm{m}$ at $4500 \mathrm{~mm} / \mathrm{s})$ there is a branched rather than only a linear crack formation over the entire heat-affected zone. We assume that, for the smallest spot spacing, the laser pulses interact with a pre-heated environment which leads to far less thermally induced mechanical strain in the dielectric layer than for the highest spot spacing, where every laser pulse practically interacts with the carbon layer at ambient condition. However, in the former case this condition is met only at the first edge of the carbon layer inducing the crack right above.

In the following printing step, the aqueous PEDOT:PSS ink is driven by capillary forces to the cavity and further down to the location of the removed carbon layer. Evidence for this 
assumption is given by a measurement of the respective surface energies via the contact angles at the solid-liquid interface (Table 1): whilst the PEDOT:PSS is repelled from the dielectric layer (contact angle $>30^{\circ}$ ), it considerably wets both a carbon layer and a PET surface as checked with reference samples. Assuming that the laser impact does not change the surface energy, in the light of an aqueous ink composition, the wetting directly after printing should be favored at the areas of the exposed PET surface where the carbon has been removed and not on the remaining parts of the carbon sub-layer themselves. This step resembles the wicking technology in microfluidics recently reported by Shao et al. where a Laplace pressure is utilized to fill channels with a liquid $^{\mathbf{3 1}}$.

$<$ Table 1>

Summarizing the manufacturing part, the functionality of our OECTs is in substance based on three requirements: (i) the conductive sub-layer is completely cut during the self-aligning laserablation step and (ii) a via-like channel is indirectly formed through the dielectric top-layer that (iii) is re-filled with PEDOT:PSS in the subsequent inkjet printing step. Finally, inkjet-printing of an appropriate electrolyte and lamination with a conductive Orgacon foil (cf. Experimental Section) render the manufacturing workflow complete.

$<$ Figure $6>$ 
Fig. 6 shows the typical output characteristics of a vertically stacked OECT fabricated according to our workflow model. The conductivity of the PEDOT:PSS channel is decreased by a positive gate voltage, which is a typical behaviour for OECTs operating in depletion mode /9/18/23/. The operational voltage, the (source-drain current) on/off ratio of the OECTs and the switch time are the most important parameters for the usage of OECTs in e.g. sensor applications /42/. When applying a gate voltage of $1 \mathrm{~V}$, the conductivity of the PEDOT:PSS decreases by at least one order of magnitude, switching the transistor from the ON to the OFF state. The on/off ratio of the source-drain current is the modulation factor between the source-drain currents $\mathrm{I}_{\mathrm{DS}}$ in these ON and the OFF states. As an example, for the OECT shown in Fig. 6, at an applied drainsource voltage $V_{D S}=-1 V$, the current is $I_{D S}$, ON $=-6 \times 10^{-5}$ A and $I_{D S}$, OFF $=-3 \times 10^{-6}$ A for gate voltages of 0 and $1 \mathrm{~V}$, respectively, yielding an on/off ratio of 20. Running this OECT at $\mathrm{V}_{\mathrm{DS}}=-$ $1.5 \mathrm{~V}$ and using $\mathrm{V}_{\mathrm{G}}=0 \mathrm{~V}\left(\mathrm{I}_{\mathrm{DS}, \mathrm{ON}}=-8 \times 10^{-5} \mathrm{~A}\right)$ and $\mathrm{V}_{\mathrm{G}}=1.5 \mathrm{~V}\left(\mathrm{I}_{\mathrm{DS}}\right.$, OFF $=-8 \times 10^{-7}$ A) to switch it, the on/off ratio reaches a value of 100. The highest on/off ratio recorded in our samples was 600 for a number of OECTs fabricated at a laser scan speed of $3500 \mathrm{~mm} / \mathrm{s}$. However, these OECTs reached saturation already at a drain-source voltage of $-0.4 \mathrm{~V}$ and incurred a drop in the on/offratio when increasing the voltage further (cf. Supporting Information).

The typical switching characteristics of an OECT are shown in Fig. 7. The drain current $\mathrm{I}_{\mathrm{DS}}$ switches off within several $100 \mathrm{~ms}$ when a gate voltage of $1 \mathrm{~V}$ is applied. In Table 2 , we have outlined the switching times for OECTs with respect to reconnection efficiency: not only does the latter (seen from an ohmic resistance in the order of $20 \mathrm{k} \Omega$ ) improve the amplitude of the current signal, an effective reconnection is also related to shorter switching times. We also see the usual effect that the ON switching times are 2-4 times longer than the OFF switching times. Here, the off-to-on (on-to-off) switch time was determined as the time it takes for the source-drain current 
to increase (decrease) from $10 \%(90 \%)$ to $90 \%(10 \%)$ upon a change of the applied gate voltage. ${ }^{\mid 9 / 42 /}$

$<$ Figure 7>

$<$ Table 2>

Finally, the integrated gate current $\mathrm{I}_{\mathrm{G}}$ over one switching event can give an insight into how the volume of PEDOT:PSS is involved in electrochemical switching in the formed channel. From the work of Andersson et al. ${ }^{150 /}$ on active-matrix displays we know about the particular electrochemistry of PEDOT:PSS upon switching the gate voltage $\mathrm{V}_{\mathrm{G}}$ : in the pristine state, the PEDOT is partly oxidized and charge neutrality is maintained by PSS $^{-}$counter ions. When a (gate) voltage is applied to a PEDOT:PSS film, the PEDOT in the negatively biased region becomes reduced, while the PEDOT in the positively biased region becomes further oxidized (“electrochemical switching"). In its oxidized state, PEDOT has a high concentration of free charge carriers and is nearly transparent in the visible range. In contrast, the reduced neutral form of PEDOT acts as an organic semiconductor with a strong absorption in the visible range. These considerations have certain implications for the reconnection step. On the one hand, the deposited volume of PEDOT:PSS ink has to secure the formation of the channel between the source and drain electrodes: wetting the carbon electrodes and the delaminated part as well as filling all the cavities in the dielectric top-layer originating from the laser-ablation step. Based on knowledge of footprint (ca. $\left.150 \times 100 \mu \mathrm{m}^{2}\right)$ and height $(3 \mu \mathrm{m})$ of the removed carbon layer (cf. Supporting Information) and an idea about the volume capacity of the formed cavity, a rough estimate on the minimum amount of PEDOT:PSS necessary to build the channel yields a value of $0.03-0.06 \mu 1$. 
However, the registration issues require that PEDOT:PSS is printed in some excess on top of the laser-induced crack, which we call overfill printing. In the light of these arguments, it turns out that inkjet printing is the method of choice as it handles individual drops of liquid in the range of $10 \mathrm{pl}$ at a deposition precision of $5 \mu \mathrm{m}$. When printing in multi-nozzle mode with a drop space of typically $20 \mu \mathrm{m}$, the printing is performed in seconds. On the other hand, since electrochemical devices rely on bulk switching of the active material, too large volume of available bulk liquid will have a detrimental impact on the switching time. Hence the overfill printing must be restricted to an inactive volume compensating the registration issues, which in the best case is electrically isolated from the PEDOT:PSS that forms the channel.

In order to probe the amount of PEDOT:PSS involved in the electrochemical switching events, Fig. 8 shows the development of the gate current $I_{G}$ in comparison to a switching of the gate voltage $\mathrm{V}_{\mathrm{G}}$ for (i) pipetted and (ii) inkjet-printed PEDOT:PSS, respectively. In the absolute current-voltage curve, the area under the curve indicates the actual amount of PEDOT involved in the electrochemical reaction, which amounts to $5.3 \times 10^{-6}$ As for pipetted and $0.4 \times 10^{-6}$ As for inkjet-printed PEDOT:PSS combined with capillary filling. This more than 12-fold difference implies that inkjet plus capillary filling is a much better choice as it deposits the PEDOT:PSS exactly where it indeed participates in the current modulation of the transistor channel. The nominal deposition volumes (pipette: $1.0 \mu 1$, inkjet: $0.17 \mu 1$ ) indicate here only a factor of 5 . The achieved drain-source currents did not significantly differ $\left(I_{D S}=-5 \times 10^{-5} A\right)$.

$<$ Figure $8>$ 


\section{Conclusions and Outlook}

In conclusion, we have shown proof of principle that the fabrication of electrochemical transistors operating at $1 \mathrm{~V}$ with switching times around $100 \mathrm{~ms}$ is feasible by a combination of semiautomatic screen printing, scanning laser ablation, inkjet printing, and foil lamination. The presented manufacturing strategy is fully compatible with standard package printing. The registration precision (placement of the batch sheets) between the individual steps is in the order of millimeters. Hence, no expensive positioning equipment is required, and manufacturing is even feasible in semiautomatic or manual processes. Due to the employed concept of overfilling, our approach is insensitive to a number of typical failures that may occur along a printed electronics value chain: clogging of individual mesh cells, variation of laser intensity in the range of several per cent, clogging of individual nozzles during inkjet printing. In our manufacturing strategy, the inclusion of digital techniques (scanning laser, inkjet printing) enables fabrication concepts for personalized devices, e.g. arrays of OECTs with varying electrolytes as the active elements in sensor components, fully compatible with standard contemporary package printing.

The total manufacturing yields were $20-25 \%$, a value that is mainly dependent on the efficiency of disconnection by the laser-ablation step and the reconnection upon the formation of a channel via capillary forces by inkjet deposition of PEDOT:PSS. While, undoubtedly, these values do not yet fully comply with industrial demands, we point out that all steps were carried out with machines available or accessible to a regular print house. With a fine tuning of the laser parameters (wavelength, pulse duration) with respect to the particular stack materials and minor improvements of the layer homogeneities we expect that much higher yields are feasible. 


\section{Experimental Section}

The samples were printed at Printed Electronics Arena (Norrköping, Sweden) using a semiautomatic screen printer (Model SCF-550, TIC Technical Industrial Co. Ltd.) and thermal and UV-based drying systems, respectively. Polyfoil (size 510x400 mm², thickness $125 \mu \mathrm{m}$; Policrom Screens, Carvico, Italy; Lot No. 00158-223) was used as substrate. To minimize alignment problems, especially during the thermal drying steps between the printing passes, the foil substrates were preshrunk in a ventilated oven at $130-140{ }^{\circ} \mathrm{C}$ for several minutes prior to printing. The source-drain structures and the dielectric layer were deposited in two individual passes with commercially available material. For the conductive layer (source-drain structure), a carbon paste (DuPont 7102; Lot No. TFP152) was mixed with a thinning liquid (DuPont 3610; Lot No. UIPE85) in the ratio 9:1. For the dielectric layer, a UV-sensitive and cross-linkable material (DuPont 5018; Lot No. THP253) was used and printed as received. First, the conductive layer was printed with one screen (mesh $120 \mathrm{~cm}^{-1}$, thread diameter $34 \mu \mathrm{m}$ ) and dried in a conveyor-belt-fed drying system (Model 410-60, Texair American Screen Printing Equipment Ltd.) at $110{ }^{\circ} \mathrm{C}$ for ca. 2.5 mins. Second, the dielectric layer was printed with a second screen having the same parameters and cured in a conveyor-belt UV dryer (Aktiprint T28-1, Technigraf) at $164 \mathrm{~mJ} / \mathrm{cm}^{2}$ for ca. 2 seconds. The screen printing parameters were set (doctor blade angle: 5 degrees, squeegee hardness: 75/95/75) and adjusted in detail with several dummy substrates until a homogeneous deposition was reproducibly achieved. Besides a visual homogeneity of the printed structures, the criterion for homogeneity was the observation that the conductivity of the individual source-drain structures was in the range of $10+/-5 \mathrm{k} \Omega$ measured with a standard digital multimeter (Model UNI58B, UNI-T). This value was determined for a number of sourcedrain structures (components) on the DIN A4 substrate having test pads separated by $1 \mathrm{~cm}$. 
For the laser treatment, an industrial laser patterning setup (microSTRUCT 1060, 3d-micromac AG, Chemnitz, Germany) was used. The machine is particularly designed for sheet-fed laser treatment of large-area substrates for printed and polymer electronics devices. It consists of a conveyor belt for the transport of the printed sheets and a closet for laser machining with an area of operation of $700 \times 700 \mathrm{~mm}^{2}$. Alignment of the fed sheets is done by an optical inspection system. The plant uses a compact and air-cooled pulsed Ytterbium Fiber Laser (IPG Laser $\mathrm{GmbH}$, Burbach, Germany) with an average power of $30 \mathrm{~W}$ at a wavelength of $1064 \mathrm{~nm}$, a maximum repetition rate of $200 \mathrm{kHz}$ and nominal pulse duration of $100 \mathrm{~ns}$. A bendable, metalcoated fiber guides the laser beam into the closet. The positioning of the laser beam on the sample is carried out with a mirror-based long-distance scanner system (focal length $820 \mathrm{~mm}$; SCANLAB AG, Puchheim, Germany). A PC interface card controls the synchronous and continuous operation of the mirrors and the laser in real time via an automation code, and this option was used to control the movement of the scanner during laser operation (mark speed) and hence the distances of the laser pulses incident on the substrate. The other relevant laser parameters were kept constant (wavelength $1064 \mathrm{~nm}$, repetition rate $200 \mathrm{kHz}$, average power 30 W). Disconnection of the source-drain structures was verified by a digital multimeter (see above). Inkjet printing was performed using a Dimatix DMP-2800 printer (Fujifilm Dimatix Inc., Santa Clara, USA). The printing setup consisted of several piezoelectric inkjet printheads with a nozzle diameter of $21.5 \mu \mathrm{m}$ and a nominal drop volume of $10 \mathrm{pl}$. All samples were printed at ambient conditions $\left(22.5 \pm 0.8{ }^{\circ} \mathrm{C}\right.$ and $40 \pm 3 \%$ relative humidity). The DMP-2800 was applied in multinozzle mode (firing frequency $2 \mathrm{kHz}$, meniscus set-point $5.0 \mathrm{mmHg}$, nozzle temperature $30^{\circ} \mathrm{C}$ ), with a clear distance between the nozzle and the substrate maintained at $1 \mathrm{~mm}$. The PEDOT:PSS ink was prepared from commercially available Clevios HC Jet P (Lot No. JOF6903-6b; H.C. Starck GmbH, Leverkusen) mixed with deionized water (ca 9:1) and treated with ultrasound (5 
min, $40{ }^{\circ} \mathrm{C}$ ) prior to filling the printing cartridges. The printing pattern was designed to overfill the particular cross section and to yield sufficient reconnection (pattern area $2 \times 2 \mathrm{~mm}^{2}$, drop space $15 \mu \mathrm{m}$, nominal film volume $0.17 \mu \mathrm{l})$. After printing, the samples were dried at $100{ }^{\circ} \mathrm{C}$ for a few seconds. A polycationic liquid with mobile anions was chosen as the electrolyte, formulated to an aqueous ink and printed with an overfill (pattern area $3 \times 3 \mathrm{~mm}^{2}$, drop space $20 \mu \mathrm{m}$, nominal film volume $0.23 \mu \mathrm{l}$ ) under similar conditions as the PEDOT:PSS ink. Alternatively, manual deposition of microdroplets (Finnpipette Focus, Thermo Scientific Inc., volume $1 \mu \mathrm{l}$ ) were applied. A commercial PEDOT:PSS-coated plastic foil (Orgacon EL-350, AGFA-Gevaert NV, Belgium; total thickness 125-175 $\mu$ m; thickness of PEDOT:PSS layer $200 \mathrm{~nm}$ ) was applied as the gate electrode on top of the solidified electrolyte layer. The pieces were cut on a sheet-fed cutterplotter (knife apex: 10-25 $\mu \mathrm{m}$ ) and placed with the conductive side in contact with the printed electrolyte. Reasonable mechanical stability of the device was achieved once the electrolyte was semi-dried, but to allow for a robust handling during the device characterization, the gate electrode was additionally fixed with tape.

The deposited layers were characterized using optical microscopy (Nikon SMZ1500, magnification 2-10x, and the built-in fiducial camera in the DMP-2800), optical profilometry (SensoFAR neox 3D Optical Profiler), and scanning electron microscopy (SEM) (JEOL JSM6335F). For cross-sectional SEM imaging of the dielectric and semiconducting interfaces, the samples were sputtered with a noble metal layer (Au, thickness ca. $3 \mathrm{~nm})$. The contact angle measurements were done in air (CAM 200 goniometer). The reconnection of the source-drain structures with inkjet-printed PEDOT:PSS was verified with a digital multimeter (see above). The current-voltage characteristics of the entire components were recorded by a parameter analyzer (Model 4155B, Agilent/HP) equipped with manual probe heads (Model PH100, Süss, apex diameter: $25 \mu \mathrm{m}$ ). The individual transistor components were cycled in the range $\mathrm{V}_{\mathrm{DS}}$ from 
0 to $-1.5 \mathrm{~V}$ with the gate voltage $\mathrm{V}_{\mathrm{G}}$ varied in steps between 0 and $1.5 \mathrm{~V}$ with an increment of $0.25 \mathrm{~V}$. The time-dependent current switching characteristics were obtained by coupling two digital sourcemeters (Keithley 2400), one of which was operating as a waveform generator and the other as a measurement device.

\section{Acknowledgements}

This project was initiated in a research collaboration between Linköping University, Acreo AB and Chemnitz University of Technology within the EU-FP7-Network of Excellence PolyNet (2008-2010), funded by the European Community's Seventh Framework Programme (FP7, 20072013) under grant agreement no. 214006 and finished in a postdoctoral scholarship issued by Linköping University, Department of Science and Technology (T. B., number ITN-2010-00018), in collaboration with PEA Manufacturing (Printed Electronics Arena, Norrköping, Sweden). The authors thank Marie Nilsson (Acreo AB) for experimental support at PEA Manufacturing, Amal Hansson (Linköping University) for assistance with the SEM measurements and Andrea Jauss, Elena Bailo and Jan Toporski (WiTec GmbH, Ulm, Germany) for performing the depth-profile confocal Raman microscopy. 


\section{References}

/1/ A. Javey, J. Guo, D. B. Farmer, Q. Wang, D. Wang, R. G. Gordon, M. Lundstrom, H. Dai, Nano Lett. 2004, 4 (3), 447-450.

/2/ R. T. Weitz, U. Zschieschang, F. Effenberger, H. Klauk, M. Burghard, K. Kern, Nano Lett. 2007, 7 (1), 22-27.

/3/ H. Klauk, U. Zschieschang, J. Pflaum, M. Halik, Nature (London) 2007, 445, 745.

/4/ M. J. Panzer, C. D. Frisbie, J. Am. Chem. Soc. 2005, 127 (19), 6960-6961.

/5/ M. J. Panzer, C. D. Frisbie, J. Am. Chem. Soc. 2007, 129 (20), 6599-6607.

/6/ E. Said, P. Andersson, I. Engquist, X. Crispin and M. Berggren; Org. Electronics 2009, 10, 1195-1199.

/7/ L. Herlogsson, M. Cölle, S. Tierney, X. Crispin, M. Berggren; Adv. Mater. 2010, 22, 72-76.

/8/ L. Herlogsson, X. Crispin, S. Tierney, M. Berggren, Adv. Mater. 2011, 23, 4684-4689

19/ D. Nilsson, M. Cheng, T. Kugler, T. Remonen, M. Amgarth, M. Berggren; Adv. Mater. 2002, 14 (1), 51-54.

/10/ D. Nilsson, N. Robinson, M. Berggren, R. Forchheimer, Adv. Mater. 2005, 17, 353-358.

/11/ O. Yaghmazadeh, F. Cicoira, D. A. Bernards, S. Y. Yang, Y. Bonnassieux, G. G. Malliaras J. Pol. Sci. B: Polymer Physics 2011, 49, 34-39.

/12/ R. Havener, J. Boyea, E. Malone, D. A. Bernards, J. Defranco, Materials Science 2001, 1, 14.

/13/ Z. T. Zhu, J. T. Mason, C. Zhu, N. C. Cady, C. A. Batt, G. G. Malliaras, Chem. Commun. 2004, 13, 1556 .

/14/ J. T. Mabeck, J. A. Defranco, D. A. Bernards, G. G. Malliaras, S. Hocdé, C. J. Chase, Appl. Phys. Lett. 2005, 87, 13503. 
/15/ J. W. Thackaray, H. S. White, M. S. Wrighton; J. Phys. Chem. 1985, 89, 5133.

/16/ V. Rani, K. S. V. Santhanam, J. Solid State Electrochem. 1998, 2, 99.

/17/ V. Saxena, V. Shirodkar, R. Prakash, J. Solid State Electrochem. 2000, 4, 234.

/18/ P. Andersson, R. Forchheimer, P. Tehrani, M. Berggren; Adv. Funct. Mater. 2007, 17, 3074-3082.

/19/ R. Mannerbro, M. Ranlöf, N. Robinson, R. Forchheimer, Synth. Metals 2008, 158, 556.

/20/ N. Kaihovirta, T. Mäkelä, X. He, C.-J. Wikman, C.-E. Wilén, R. Österbacka, Org. Electronics 2010, 11, 1207.

/21/ M. Hamedi, R. Forchheimer, O. Inganäs, Nature Materials 2007, 6, 357.

/22/ C. Müller, M. Hamedi, R. Karlsson, R. Jansson, R. Marcilla, M. Hedhammar, O. Inganäs, Adv. Mater. 2011, 23, 898-901.

/23/ M. Zirkl, A. Sawatdee, U. Helbig, M. Krause, G. Scheipl, E. Kraker, P. Andersson Ersman, D. Nilsson, D. Platt, P. Bodö, S. Bauer, G. Domann, B. Stadlober; Adv. Mater. 2011, 23, 20692074.

/24/ A. C. Hübler, G. C. Schmidt, H. Kempa, K. Reuter, M. Hambsch, M. Bellmann, Org. Electronics 2011, 12 (3), 419-423.

/25/ F. C. Krebs, Solar Energy Materials and Solar Cells 2009, 93 (4), 394-412.

/26/ T. Todorov, J. Mitzi, Eur. J. Inorg. Chem. 2010, 1, 17-28.

/27/ T. Fischer, U. Hahn, M. Dinter, M. Bartzsch, G. C. Schmidt, H. Kempa, A. C. Hübler; Org. Electronics 2009, 10, 547-550.

/28/ S. F. Jahn, A. Jakob, T. Blaudeck, P. Schmidt, H. Lang, R. R. Baumann; Thin Solid Films 2010, 518, 3218-3222.

/29/ S. F. Jahn, T. Blaudeck, R. R. Baumann, A. Jakob, P. Ecorchard, T. Rüffer, H. Lang, P. Schmidt; Chem. Mater. 2010, 22 (10), 3067-3071. 
130/ H.-Y. Tseng, B. Purushothaman, J. Anthony, V. Subramanian, Org. Electronics 2011, 12, 1120-1125.

/31/ F. Shao, T. N. Ng, J. Fu, W. Shen, W. Y. L. Ling, J. Coll. Interf. Sci. 2011, 363, 425-430.

132/ S. H. Ko, J. Chung, H. Pan, C. P. Grigoropoulos, D. Poulikakos; Sensors and Actuators A 2007, 134, 161-168.

/33/ C.-Y. Lo, J. Hiitola-Keinänen, O.-H. Huttunen, J. Petäjä, J. Hast, A. Maaninen, H. Kopola, H. Fujita, H. Toshiyoshi; Microelectronic Engineering 2009, 86, 979-983.

/34/ N. G. Semaltianos, C. Koidis, C. Pitsalidis, P. Karagiannidis, S. Logothetidis, W. Perrie, D. Liu, S. P. Edwardson, E. Fearon, R. J. Potter, G. Dearden, K. G. Watkins; Synth. Metals 2011, $161,431-439$.

/35/ A. Schoonderbeek, V. Schütz, O. Haupt, U. Stute; J. Laser Micro Nanoeng. 2010, 5, 248255.

/36/ R. R. Baumann, A. Willert, T. Blaudeck; Adv. Printing and Media Technology 2010, 37, 327-334.

/37/ F. C. Krebs, Org. Electronics 2009, 10, 761-768.

/38/ F. C. Krebs, J. Fyenbo, M. Jørgensen; J. Mater. Chem. 2010, 20, 8994-9001.

/39/ J. Liu, L. Herlogsson, A. Sawatdee, P. Favia, M. Sandberg, X. Crispin, I. Engquist, M. Berggren; Appl. Phys. Lett. 2010, 97, 103303.

/40/ M. Jung, J. Kim, J. Noh, N. Lim, C. Lim, G. Lee, J. Kim, H. Kang, K. Jung, A. D. Leonard, J. M. Tour, G. Cho, IEEE Transactions on Electron Devices 2010, 57 (3), 571580.

/41/ P. Tehrani, I. Engquist, N. D. Robinson, D. Nilsson, M. Robertsson, M. Berggren, Electrochim. Acta 2010, 55, 7061-7066. 
/42/ M. Berggren et al.: "PEDOT:PSS-Based Electrochemical Transistors for Ion-to-Electron Transduction and Sensor Signal Amplification", in: D. A. Bernards, R. M. Owens, G. G. Malliaras (eds.): “Organic Semiconductors in Sensor Applications”, Springer-Verlag, 2008. /43/ A. Ziegler, MRS Bulletin 2011, 36, 121-131.

/44/ T. Szörényi , Z. Kántor, L. D. Laude, Appl. Surf. Sci. 1995, 86, 219.

/45/ A. I. Kuznetsov, J. Koch, B. N. Chichkov, Opt. Express 2009, 17 (21), 18820.

/46/ U. Schmidt, A. Jauss, W. Ibach, K. Weishaupt, O. Hollricher, Microscopy Today 2005, 5, 30-34.

/47/ C. A. Michaels, J. Raman Spectroscopy 2010, 41, 1670-1677.

/48/ A. N. Aleshin, R. Kiebooms, R. Menon, F. Wudl, A. J. Heeger, Phys. Rev. B 1997, 56 (7), 3659-3663.

/49/ A. N. Aleshin, S. R. Williams, A. J. Heeger; Synth. Metals 1998, 94, 173-177.

150/ P. Andersson, D. Nilsson, P. Svensson, M. Chen, A. Malmström, T. Remonen, T. Kugler M. Berggren, Adv. Mater. 2002, 14, 1460-1464. 


\section{Figures}

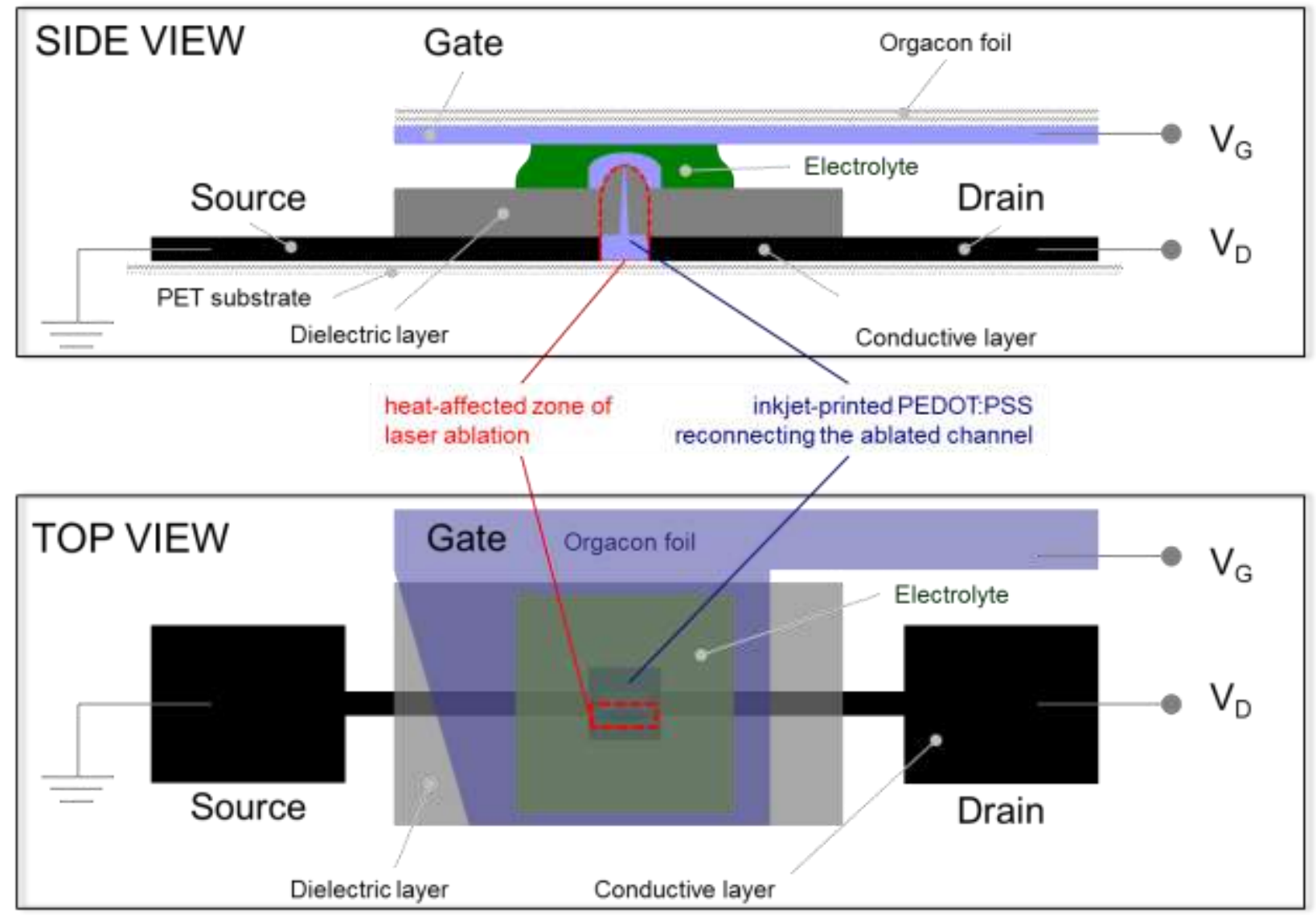

Figure 1: Side view and top view of the vertical stack to build an individual organic electrochemical transistor (OECT) by printing and laser ablation. 

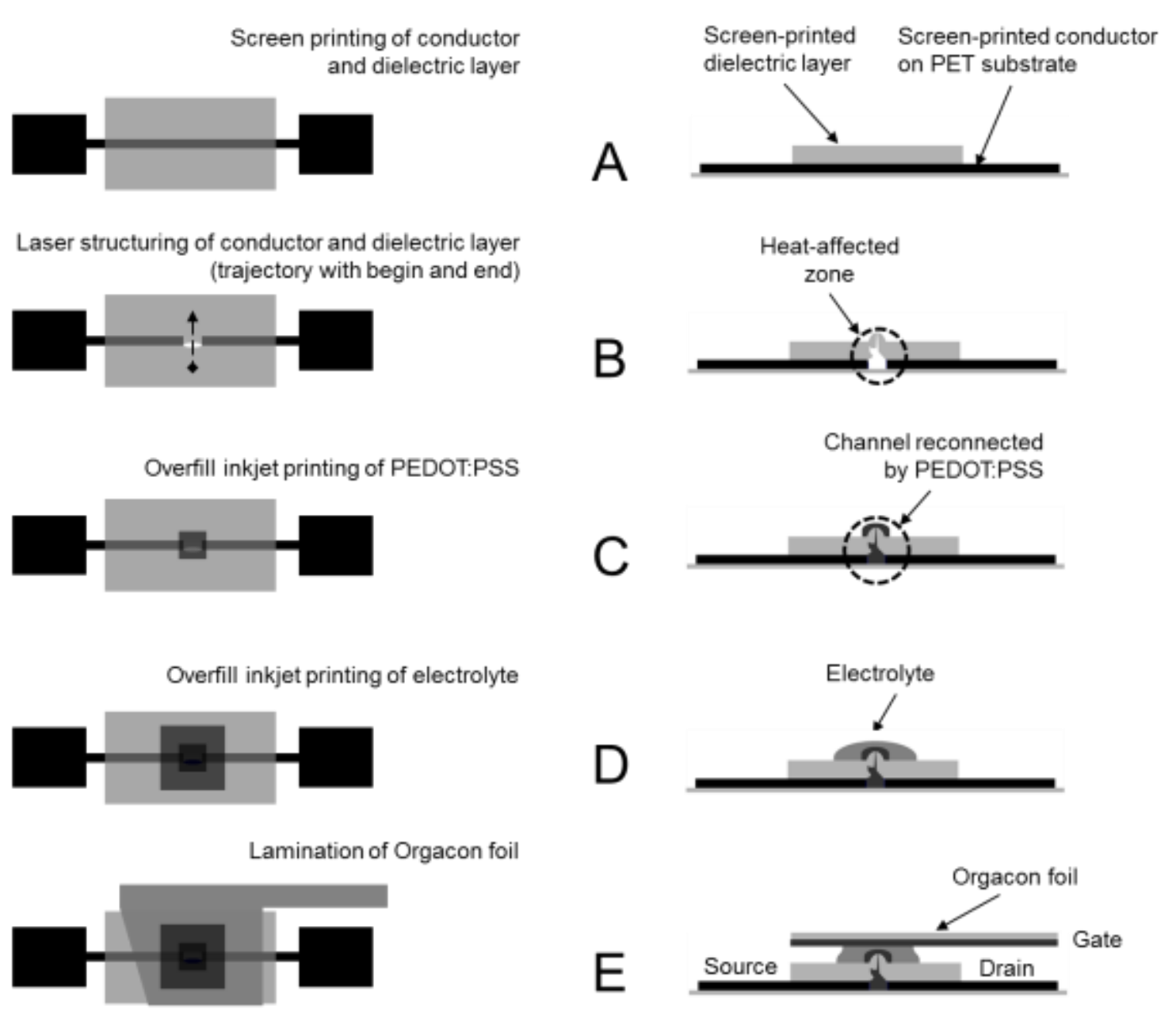

Figure 2: Hybrid manufacturing scheme for printed electrochemical transistors (ECTs) (side view): (A) two subsequent passes of screen printing: one conductive source-drain structure and one dielectric layer; $(B)$ laser structuring results in a disconnection of the source-drain structure as well as an indirect perforation of the transparent dielectric layer; the achieved active channel areas are of the order $0.02 \mathrm{~mm}^{2} ;(C)$ the local destruction of the dielectric layer after laser treatment in $(B)$ allows a reconnection of the transistor channel via overfill printing of PEDOT:PSS (inkjet); (D) overfill printing of the electrolyte (inkjet); (E) lamination of a cut-out PEDOT:PSS-coated PET foil, Orgacon EL-350, as the top gate electrode. During the entire workflow, a manual positioning and orientation of a DIN A4 substrate sheet in the range of millimeters and higher is sufficient. 


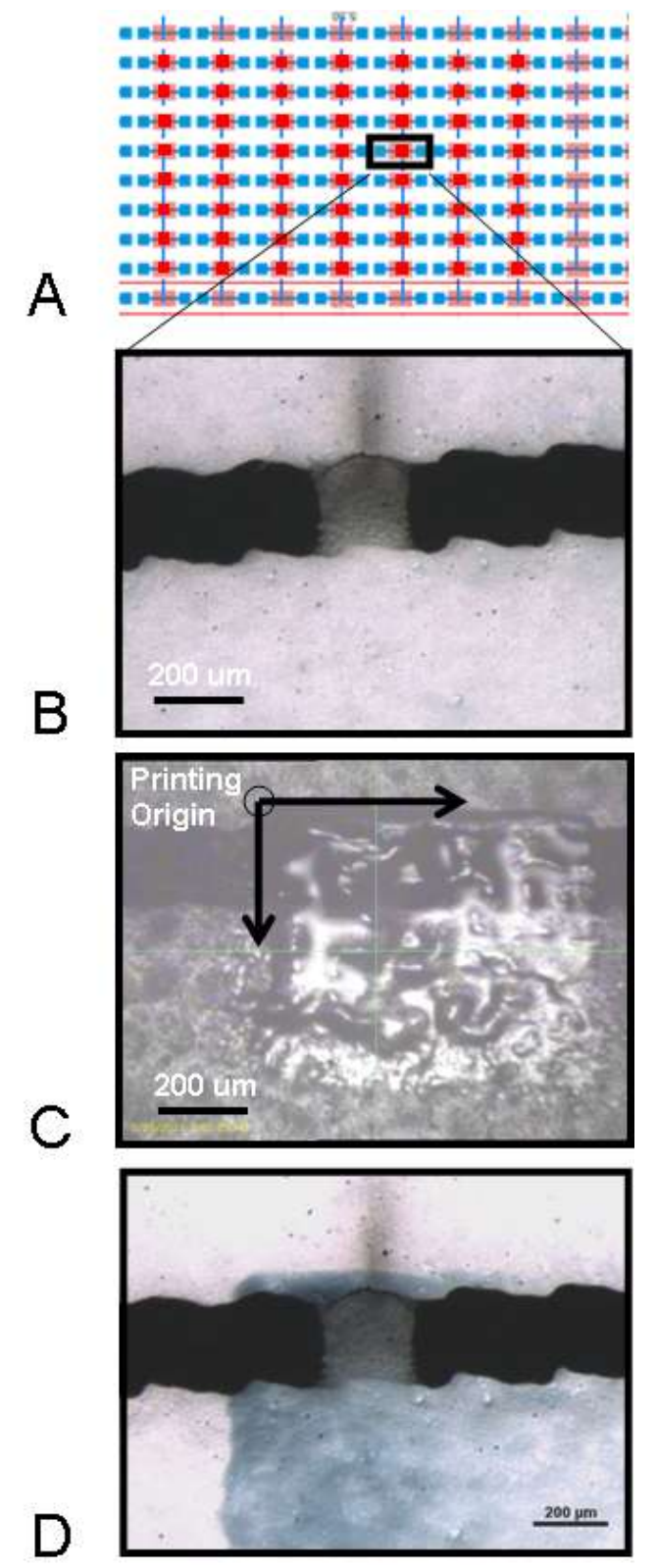

Figure 3: (A) Part of the original computer-aided design (CAD) drawing for manufacturing of an OECT array. It contains the conductive source-drain structure (blue "dogbones"), the dielectric top-layer (light red rectangle). The laser trajectories (vertical blue lines) and printing areas for PEDOT:PSS (red squares) are indicated. (B-D) Optical microscope images of (B) the channel region after laser ablation, $(C)$ directly after reconnection with overfill-printing of PEDOT:PSS, and (D) after drying of the overfill-printed PEDOT:PSS. 


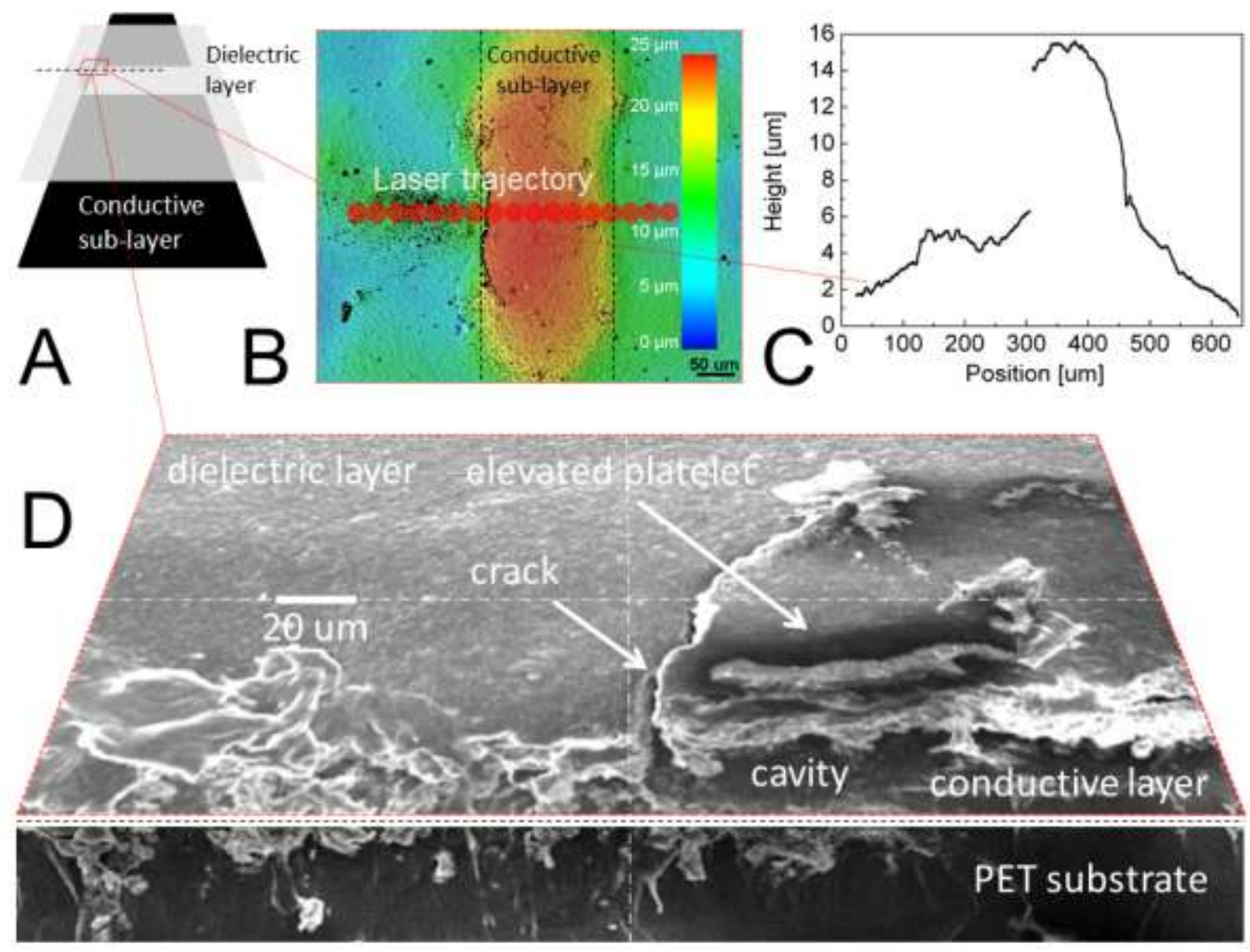

Figure 4: (A) Principal sketch of the channel region. (B) Optical profilogram and $(C)$ vertical profile scan showing an elevated plateau of the dielectric layer in the area of the heat affected zone. The red circles indicate the separated laser spots (diameter ca. $150 \mu \mathrm{m}$ ) that form a trajectory, the black pixels in the profilogram and the missing data in the curve indicate inaccessible slopes in the topography, assigned to the crack of 1-2 $\mu \mathrm{m}$ width. (D) Cross-sectional SEM showing that the laser ablation forms an elevated platelet of the dielectric layer which creates a cavity. This cavity is reconnected by inkjet-printed PEDOT:PSS. 


\section{Relative Occurrence [\%]}

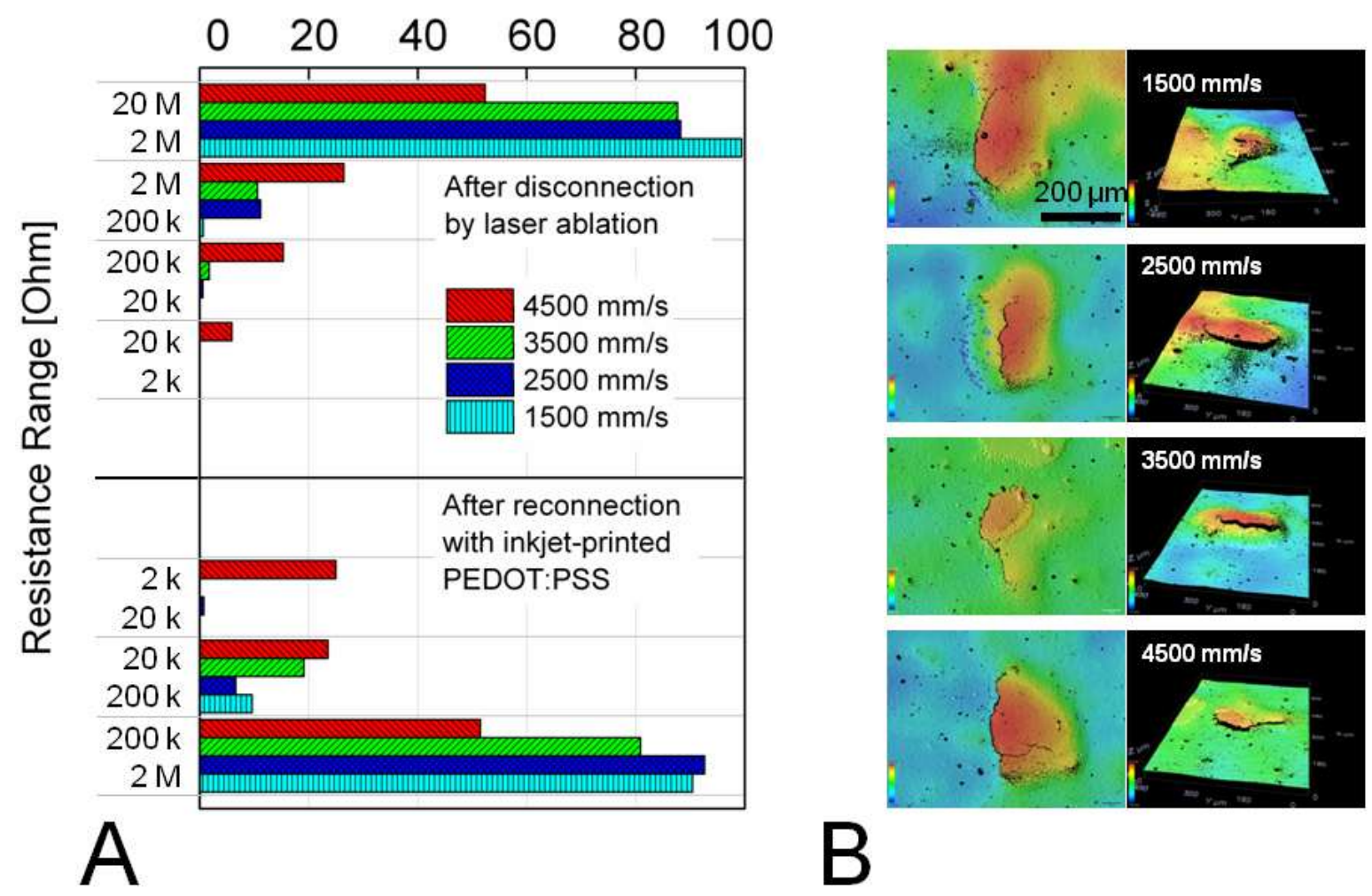

Figure 5: (A) Manufacture yields of the disconnection step by laser ablation (upper part) and the reconnection step by overfill-printing of PEDOT:PSS (lower part) as a function of the scanning speed of the laser beam. The values on the vertical axis indicate the upper and lower resistance values characterizing the respective batch of source-drain structures. (B) Optical profilograms of typical "cracks" formed as a function of the laser scan speed applied during the disconnection step. 
Table 1: Contact angle $\left(^{\circ}\right)$ of the ink constituents (PEDOT:PSS : deionized water @ 9:1) with respect to the involved layers.

\begin{tabular}{|l|l|l|l|}
\hline Layer & $\begin{array}{l}\text { PET substrate } \\
\text { (Polyfoil) }\end{array}$ & $\begin{array}{l}\text { Conductive } \\
\text { (DuPont 7102: } \\
\text { DuPont 3610 @ 9:1) }\end{array}$ & $\begin{array}{l}\text { Dielectric } \\
\text { (DuPont 5018) }\end{array}$ \\
\hline Deionized water (DI) & $29+/-7$ & $105+/-5$ & $89+/-2$ \\
\hline $\begin{array}{l}\text { PEDOT:PSS } \\
\text { (Clevios HC Jet P) }\end{array}$ & $10+/-5$ & $10+/-5$ & $31+/-9$ \\
\hline
\end{tabular}




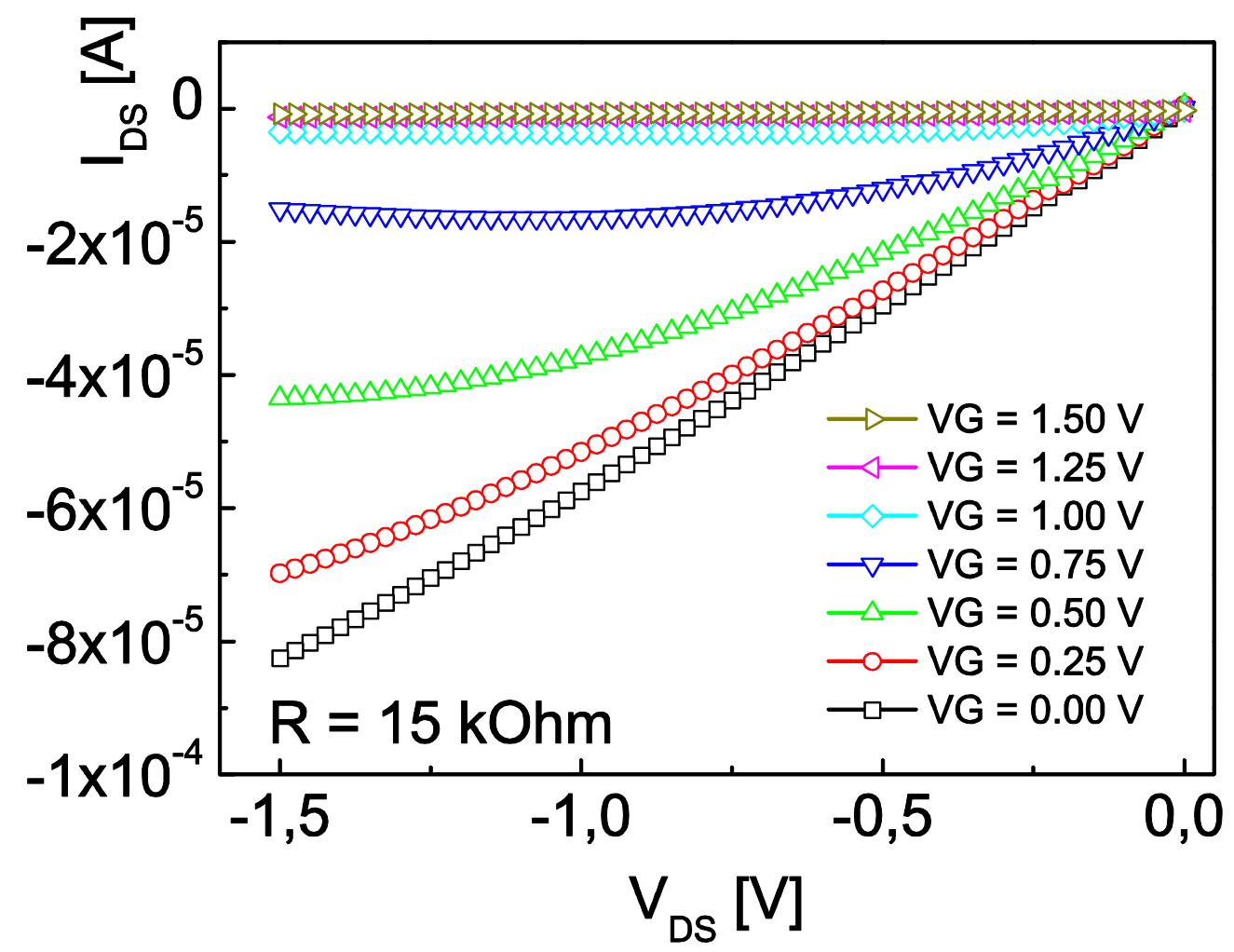

Figure 6: Output characteristics of a typical OECT fabricated via the hybrid manufacturing approach showing the drain-source current $I_{D S}$ with respect to the drain-source voltage $V_{D S}$. The gate voltage $V_{G}$ was varied from 0 to $1.5 \mathrm{~V}$. 


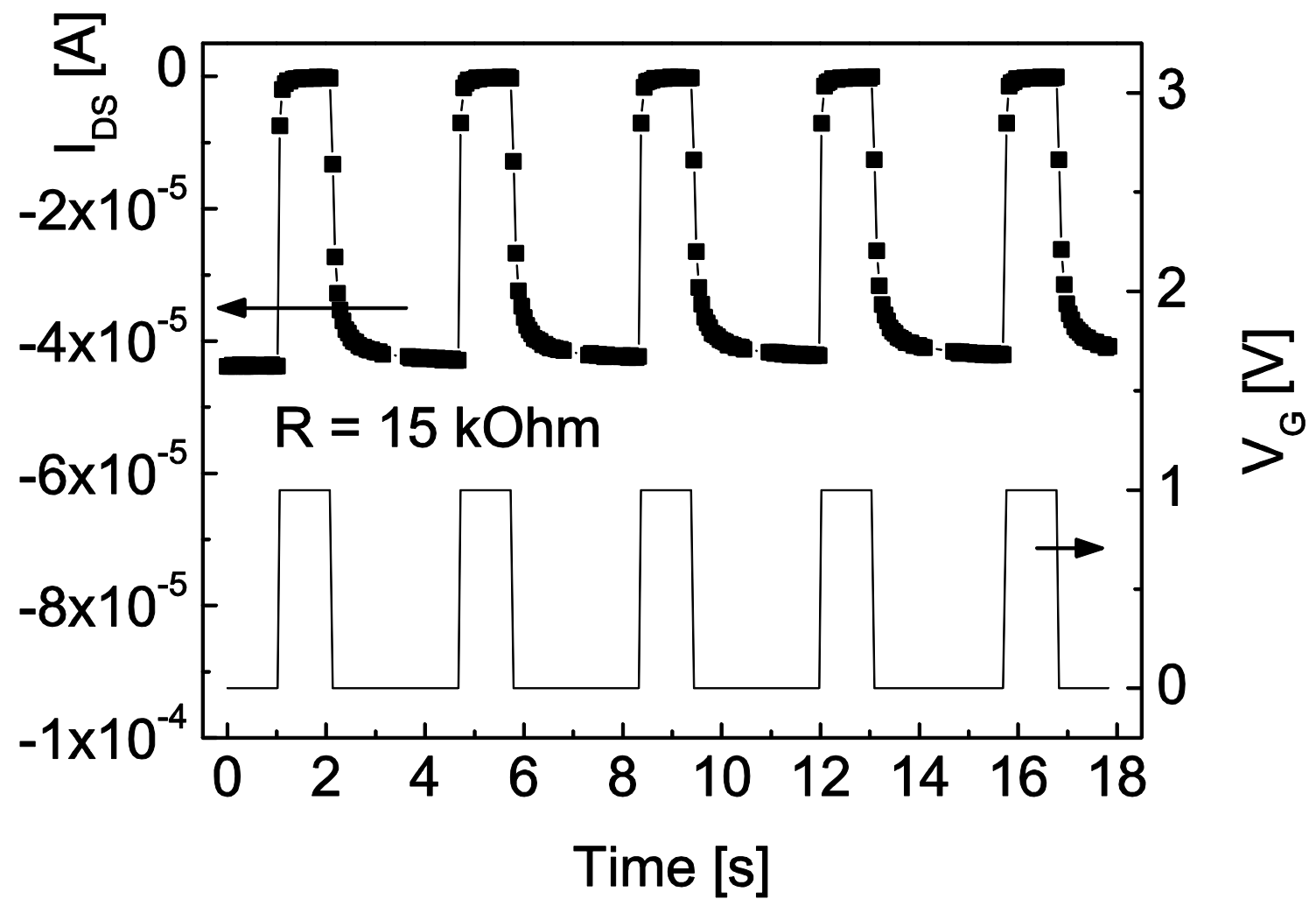

Figure 7: Switching characteristics of a typical OECT fabricated via the hybrid manufacturing approach showing $I_{D S}$ as a function of time during repeated switching of $V_{G}$ between 0 and $1 \mathrm{~V}$ at a drain-source voltage $V_{D S}=-1 \mathrm{~V}$. 


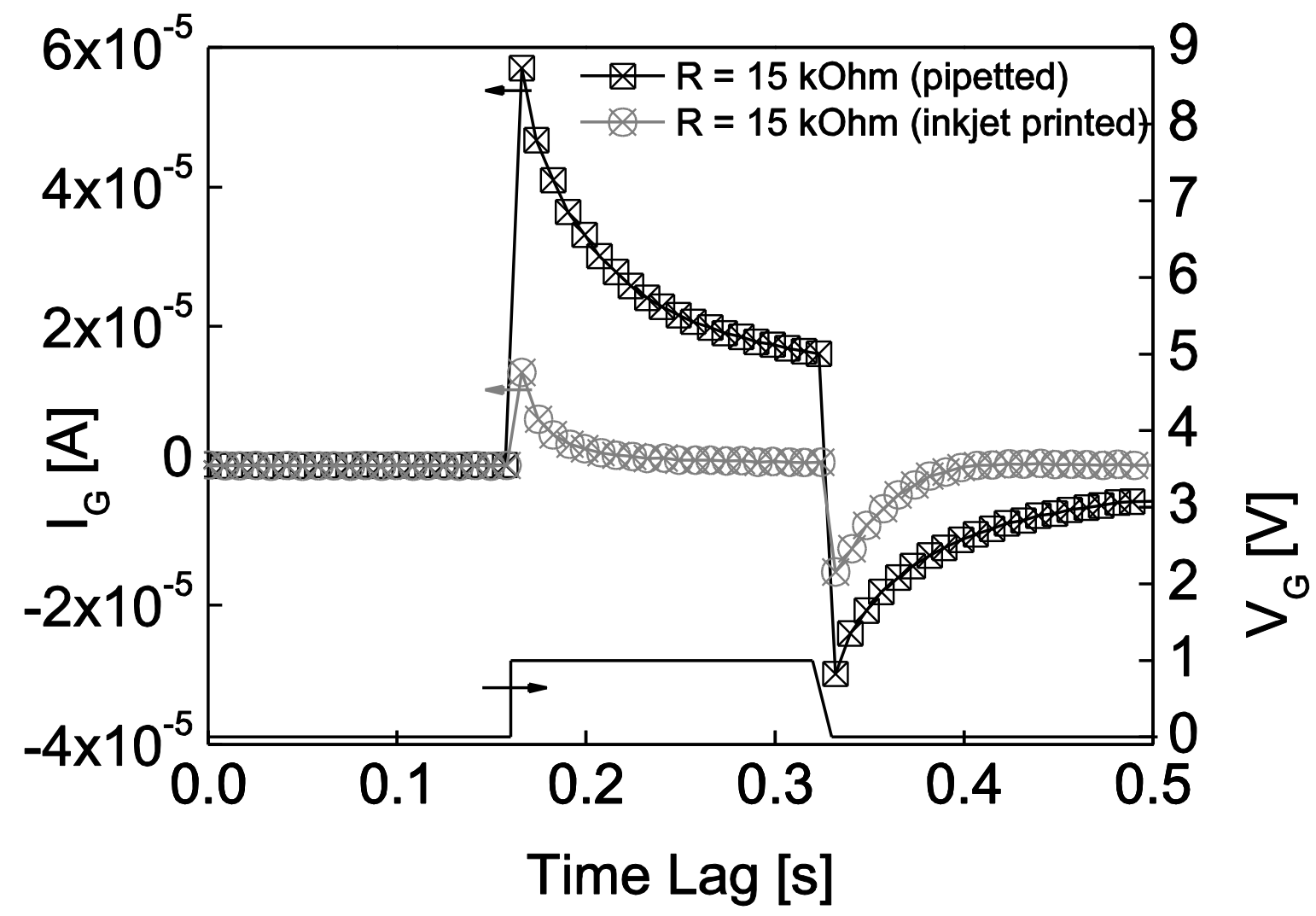

Figure 8: Response of the gate current (left axis) as a function of gate voltage switching (right axis) for pipetted (black squares) and inkjet-printed (gray circles) PEDOT:PSS. The curves were measured for OECTs of similar ohmic resistance (15 $\mathrm{k} \Omega$ ) after reconnection. 
Table 2: On-to-off and off-to-on switching times (ms) of typical OECTs as a function of the ohmic resistance of the reconnected source-drain structure after disconnection by laser ablation and reconnection by inkjet-printed PEDOT:PSS.

\begin{tabular}{|l|l|l|c|}
\hline \multicolumn{1}{|c|}{ Ohmic resistance } & \multicolumn{1}{c|}{$<32 \mathrm{k} \Omega$} & \multicolumn{1}{c|}{$32-82 \mathrm{k} \Omega$} & $>82 \mathrm{k} \Omega$ \\
\hline On-to-off $(\mathrm{ms})$ & $160+/-40$ & $360+/-60$ & $560+/-90$ \\
\hline Off-to-on $(\mathrm{ms})$ & $460+/-70$ & $860+/-130$ & $2200+/-300$ \\
\hline
\end{tabular}

